\title{
EFECTO DEL PASTOREO DE CERDOS SOBRE LAS FRACCIONES DE NITRÓGENO, CARBONO Y FÓSFORO DEL SUELO
}

\section{EFFECT OF GRAZING PIGS ON CARBON, NITROGEN AND PHOSPHORUS FRACTIONS IN SOIL}

\author{
Carmen Rivero $^{1 *}$, Eliecer Cabrales ${ }^{2}$, Giovanna Santana ${ }^{3}$, Mayra Rivas $^{3}$, Mansonia Pulido $^{1}$, \\ Juan C. Rey ${ }^{1}$, Deyanira Lobo' ${ }^{1}$ Zenaida Lozano' ${ }^{1}$, Humberto Araque ${ }^{4}$
}

Recibido para publicación: Junio 15 de 2011 - Aceptado para publicación: Marzo 4 de 2013

\begin{abstract}
RESUMEN
El objetivo del presente trabajo fue evaluar el efecto del pastoreo de cerdos, en algunas variables químicas en un Molisol en Maracay - Venezuela, bajo condiciones isohipertérmicas. Se tomaron muestras de suelos de dos lotes, uno sin cerdos y el otro con cerdos (6 $\mathrm{kg}$ de peso vivo por $\mathrm{m} 2)$. Se consideraron dos profundidades de muestreo $(0-5$ y $5-10 \mathrm{~cm}$ ) bajo un diseño factorial $2 \times 2$. Se determinó nitrógeno total, nitrógeno amoniacal, nitrógeno nítrico y nitrógeno orgánico. Se realizó la extracción y el fraccionamiento de la materia orgánica del suelo (MOS) y se determinó el carbono orgánico total oxidable, carbono extraíble total (CET), carbono unido a los ácidos húmicos (CAH), los ácidos fúlvicos (CAF) y sustancias no húmicas (CSNH). Además, se realizó el fraccionamiento del fósforo del suelo usando el método de Hedley, extrayéndose las fracciones de fósforo intercambiable en resina (P-membrana), fósforo extraído con bicarbonato de sodio, fósforo inorgánico extraíble en $\mathrm{NaOH}$, fósforo inorgánico extraíble en $\mathrm{HCl}$ y fósforo inorgánico extraíble en $\mathrm{HCl}$ caliente. Los tratamientos aplicados no afectaron el nitrógeno, el CAH y el CAF, pero si el CET y CSNH en las parcelas con cerdos. . Se observó incremento en las fracciones de fósforo evaluadas, especialmente el fósforo disponible, que fue mayor en el lote con cerdos.
\end{abstract}

Palabras clave: cerdos a campo, nitrógeno, nitrato, amonio, fósforo, carbono

\begin{abstract}
The objective of this study was to evaluate the influence of grazing pigs on some soil chemical variables in a Mollisol in Maracay - Venezuela, under isohyperthermic conditions. Soil samples were taken from grazed $(6 \mathrm{~kg} / \mathrm{m} 2)$ and non-grazed plots. Tat $(0-5$ or 5-10 cm depth), using a $2 \times 2$ factorial design. Total nitrogen, ammonia nitrogen, nitrate nitrogen and organic nitrogen were assessed. The extraction and fractionation of soil organic matter (SOM) and total oxidable organic carbon, total extractable carbon (CET), the carbon bonded
\end{abstract}

\footnotetext{
1*Ph.D. Docente, Instituto de Edafología, Facultad de Agronomía, Universidad Central de Venezuela E- mail: crivert@ewinet.com 'Docente, Instituto de Edafología, Facultad de Agronomía, Universidad Central de Venezuela

${ }^{2}$ M.Sc. Docente Área suelos, Universidad de Córdoba-Colombia. ecabralesh@yahoo.es

${ }^{3}$ Postgrado en Ciencia del Suelo, Universidad Central de Venezuela

${ }^{4}$ Instituto de Producción Animal, Facultad de Agronomía, Universidad Central de Venezuela
} 
to humic acids $(\mathrm{CAH})$, fulvic acids $(\mathrm{CAF})$, and non-humic substances $(\mathrm{CSNH})$ were determinated. In addition, the phosphorous in the soil was fractionated by using the method of Hedley; extracting the fractions: resin exchangeable phosphorus (P-membrane), phosphorus extracted with sodium bicarbonate, $\mathrm{NaOH}$ extractable inorganic phosphorus, inorganic phosphorus and inorganic phosphorus extractable by $\mathrm{HCl}$ and inorganic phosphorus extractable in hot $\mathrm{HCl}$. The treatments did not affect nitrogen, $\mathrm{CAH}$ and CAF, but the CET and CSNH were affected. . An increase on the fractions of phosphorus was observed, with higher increases in exchangeable phosphorus in grazed plots

Key words: pigs, nitrogen, nitrate, ammonium, phosphorus, carbon

\section{INTRODUCCIÓN}

El sistema de producción de cerdos a campo se caracteriza por tener a los cerdos en potreros con buena cobertura vegetal, en las fases de reproducción, maternidad e iniciación, cercados con alambres o mayas electrificadas (Dalla 1998). Estos sistemas de producción se encuentran muy distribuidos a nivel mundial puesto que se ha comprobado que tiene ciertas ventajas sobre el sistema a confinamiento tales como: bajo impacto ambiental, mayor comodidad para los animales, mejoramiento de la propiedades físicas y químicas del suelo, menor gasto en instalaciones y mano de obra para su mantenimiento, ya que se facilita el manejo del estiércol (Campagna y Somenzini 2005; Olivas y Sequeira 2002; Sosa 2005). Estos planteamientos hacen suponer que el manejo de un suelo bajo uso de cerdos a campo puede provocar modificaciones importantes de la fracción orgánica del suelo. En Venezuela se ha venido instrumentando este sistema con evaluaciones exhaustivas desde el punto de vista animal pero con muy poca o ninguna evaluación de sus efectos sobre el suelo. En general las deyecciones de los cerdos contienen minerales, especialmente micronutrimentos, que no son posibles de encontrar en los abonos comerciales (Díaz y Egaña 2000).
La dinámica del nitrógeno en el suelo se ve comúnmente afectada por el pastoreo de animales en producción, y en el caso de porcinos, los sistemas de producción de cerdos a campo son una alternativa para la fertilización. Los cerdos solo poseen la capacidad de asimilar entre 20 y $70 \%$ del $\mathrm{N}$ contenido en los alimentos lo que implica la liberación de 20 a $30 \%$ del nitrógeno en las excretas, depositándolo en el suelo en forma de urea por medio de la orina y de nitrógeno amoniacal en las heces. Al respecto Hountin et al. (1997) indican que la aplicación de purines de cerdo provoca incrementos lineales de las concentraciones de nitrógeno en el suelo, las cuales pueden alcanzar hasta los $100 \mathrm{~cm}$ de profundidad. Se plantea que el nitrógeno, bajo formas amoniacales, en estos materiales es rápidamente nitrificado, esto presentaría dos hipótesis: la disponibilidad inmediata para las plantas o la pérdida del elemento por lixiviación en una función directa a las características del suelo (Maag y Vinther 1999).

En la producción de cerdos a campo las excretas son depositadas directamente sobre el suelo, por lo cual, ofrece una mayor disponibilidad de nutrientes en la planta y reduce los efectos de la contaminación en el suelo (González 1999; Ternicier 2005). En general, los cerdos solo aprovechan entre el $50 \%$ y $60 \%$ de los 
nutrientes presente en los alimentos que consumen y es por ello, que sus excretas poseen alto contenido de nitrógeno, fósforo, potasio, calcio, magnesio, materia orgánica, entre otros, que podrían ser utilizados bien por deposición directa (pastoreo) o bien por el uso de materiales pretratados (Rochette et al. 2000; Díaz y Egaña 2000).

Ahora bien, el fósforo ha sido uno de los elementos más estudiados desde el punto de vista agrícola y aún se sigue investigando sobre ello (Navarro y Navarro 2000). El uso de sistemas de manejo que puedan aportar en forma natural estos elementos al suelo resulta de vital importancia, dados los elevados costos de la aplicación de fertilizantes sintéticos, además de su impacto en el ambiente. En este sentido, las heces de los cerdos poseen elevados contenidos de fósforo dada la poca eficiencia de estos animales para utilizar los fitatos presentes en los alimentos vegetales que consumen y ello se constituiría en un excelente aporte de este elemento (Wodzinski y Ullah 1996). Los esquemas de fraccionamiento que permitan identificar los compartimientos del suelo a los cuales se dirige un determinado elemento han sido aplicados en múltiples oportunidades, especialmente en el caso del fósforo, por cuanto ello permite indicar sobre cuál de las fracciones del elemento está incidiendo el manejo y de esta manera predecir los niveles de disponibilidad del mismo. Se ha señalado que el manejo al cual es sometido el suelo puede modificar el contenido de $\mathrm{P}$ en las fracciones y modificar la proporción entre las fracciones de menor y mayor disponibilidad (Buehler et al. 2002; Juárez et al. 2004). El objetivo de este trabajo fue evaluar el impacto del manejo de sistemas de producción de cerdos a campo sobre las distintas fracciones del nitrógeno, la materia orgánica estable y el fósforo en un suelo Molisol franco arcillolimoso de los valles de Aragua en Venezuela.

\section{MATERIALES Y MÉTODOS}

El ensayo se realizó en la sección de porcinos de la Facultad de Agronomía de la UCV en Maracay - Venezuela. El suelo corresponde a un Molisol, bajo condiciones isohipertérmicas, cuyas principales características se muestran en la tabla 1 (Rodríguez et al. 2010), destacando que es rico en bases como calcio y magnesio principalmente, el área total de estudio estuvo dividida en dos potreros de una hectárea cada uno: con cerdos a campo (6 kg de peso vivo por $\left.\mathrm{m}^{2}\right)$ y otro sin cerdos, ambos establecidos con pastos Swazi (Digitaria swazilandensis) y Angleton (Dichantium aristatum). En el potrero con cerdos, los animales permanecieron 174 días entre los años 2003 y 2006, durante los cuales comían, excretaban y dormían en el mismo.

Tabla 1. Características físicas y químicas iniciales del suelo tipo Molisol de la Facultad de Agronomía de la Universidad Central de Venezuela.

\begin{tabular}{|c|c|c|c|c|c|c|c|c|c|}
\hline & & & $\mathrm{pH}$ & & $P$ & $\mathrm{~K}$ & $\mathrm{Ca}$ & $\mathrm{Mg}$ & \\
\hline $\begin{array}{c}\mathrm{CO} \\
\text { g. } \mathrm{kg}^{-1}\end{array}$ & $\begin{array}{c}\text { CE } \\
\mathrm{dS} \cdot \mathrm{m}^{-1}\end{array}$ & $\begin{array}{c}\mathrm{CIC} \\
\mathrm{cmol}_{\mathrm{c}} \cdot \mathrm{kg}^{-1}\end{array}$ & $\begin{array}{c}1: 1 \\
\text { agua }\end{array}$ & $\begin{array}{l}\mathrm{N} \\
\%\end{array}$ & \multicolumn{4}{|c|}{$\mathrm{mg} \cdot \mathrm{kg}^{-1}$} & Textura \\
\hline 32,30 & 0,06 & 18,88 & 5,98 & 0,22 & 10,1 & 21,7 & 1380 & 155 & $\mathrm{FAL}$ \\
\hline
\end{tabular}

$\mathrm{FAL}=$ franco arcillo-limoso. Fuente: Rodríguez (2010) 
El muestreo para las evaluaciones se realizó luego de un período de descanso. Se usó un diseño completamente aleatorizado con arreglo de tratamientos del tipo factorial puro $2^{2}$, donde los factores fueron el manejo en campo (sin cerdos y con cerdos) y la profundidad (0-5 y 5-10 cm). El procesamiento estadístico de la información se realizó mediante el programa Statistix 8.0.

La determinación de Nitrógeno total se realizó mediante el método de Kjeldahl modificado de acuerdo a lo descrito por Bremner (1996). La determinación del Nitrógeno Nítrico y Amoniacal se llevó a cabo mediante una extracción con $\mathrm{KCl} 2 \mathrm{M}$ luego el $\mathrm{N}-\mathrm{NH}_{4}$ y el $\mathrm{N}-\mathrm{NO}_{3}$ se analizaron por micro Kjeldahl, sin uso y con uso de aleación Devarda (Bremner 1965). El nitrógeno orgánico fue obtenido por diferencia $\left(\mathrm{N}_{\text {ORGÁNICO }}=\mathrm{N}_{\mathrm{T}}-\mathrm{N}_{\mathrm{NH} 4}-\mathrm{N}_{\mathrm{NO} 3}\right)$. La materia orgánica fue extraída y fraccionada con el uso de una mezcla 1:1 de $\mathrm{NaOH} / \mathrm{Na}_{2} \mathrm{P}_{2} \mathrm{O}_{7}$ 0,1 M y acidificación del extracto alcalino de acuerdo a lo descrito por Rivero et al. (1998). Posteriormente para separar el carbono en las sustancias no húmicas ( $\mathrm{CSNH}$ ) se usó una columna de polivinilpilorridona sólida de acuerdo al método propuesto por Ciavatta et al. (1990). El contenido de carbono orgánico en cada fracción, extraíble total (CET), unido a los ácidos húmicos $(\mathrm{CAH})$, unido a los ácidos fúlvicos (CAF) y $\mathrm{CSNH}$ se determinó por digestión húmeda de acuerdo a lo descrito por Heanes (1984). Para el fraccionamiento del fósforo se utilizó el método de Hedley et al. (1982), modificado por Tiessen y Moir (1993) y ajustado por Salas (2001). Las fracciones evaluadas fueron: fósforo intercambiable, $\mathrm{P}$ lábil o disponible para las plantas en resina (P-membrana y $\mathrm{Pi}-\mathrm{NaHCO}_{3}$ ), fósforo inorgánico extraíble en $\mathrm{NaOH}(\mathrm{Pi}-\mathrm{NaOH})$ y que se corresponde con el $\mathrm{P}$ moderadamente disponible, fósforo inorgánico extraíble en $\mathrm{HCl}$ (Pi-HCl), fósforo inorgánico extraíble en $\mathrm{HCl}$ caliente (Pi-HCl caliente), la cual constituye una fracción de muy baja disponibilidad, fósforo inorgánico residual (P-residual) y fósforo inorgánico total (P-total). La cuantificación del $\mathrm{P}$ presente en cada fracción se hizo por el método de Murphy y Riley (1962). Complementariamente se determinó el $\mathrm{pH}$ en suspensión 1:2,5 suelo: agua en cada uno de las muestras en ambas profundidades.

Este fraccionamiento permite estimar el contenido de formas disponibles para las plantas o lábiles, el cual consiste en $\mathrm{Pi}$ en solución o adsorbido a hierro y aluminio en la matriz de intercambio (resina-Pi, $\mathrm{NaHCO}_{3}-\mathrm{Pi}$ ), el Po de rápida mineralización $\left(\mathrm{NaHCO}_{3}-\mathrm{Po}\right)$, el $\mathrm{P}$ orgánico e inorgánico moderadamente disponible o lábil asociado a fosfatos cristalinos o amorfos de hierro y aluminio $(\mathrm{NaOH}-\mathrm{Pi}$ y $\mathrm{NaOH}-\mathrm{Po})$ y las formas más estables $(\mathrm{HCl}-\mathrm{Pi}$, $\mathrm{HCl}-\mathrm{Po}, \mathrm{P}$-residual) que contiene una mezcla de fosfatos de muy baja disponibilidad para las plantas asociados a $\mathrm{Pi}$ ocluido o a minerales primarios (p.e. Pi-Ca) y Po en la materia orgánica particulada y/o protegido por la celulosa.

La fracción extraíble con $\mathrm{HCl} 1 \mathrm{M}$, con la cual se obtiene el $\mathrm{Pi}$ asociado a fosfatos de calcio (Hedley et al. 1982) y que precede a la extracción con $\mathrm{HCl}$ concentrado y caliente fue obviada en este trabajo, ya que se ha demostrado que en suelos ácidos muy meteorizados los tenores de esta fracción son muy bajas o están ausentes (Tiessen y Moir 1993). 


\section{RESULTADOS Y DISCUSIÓN}

Evaluación del Nitrógeno. Los resultados obtenidos muestran que, en términos generales, los contenidos de nitrógeno en el suelo van de medios a bajos, para las unidades experimentales estudiadas (Tabla 2).

Aun cuando los mayores contenidos de nitrógeno se encontraron en el tratamiento con cerdos a campo, dichos valores no fueron significativamente distintos. Estos resultados difieren de aquellos obtenidos por otros investigadores quienes indican que las excretas añadidas modifican sustancialmente las concentraciones de algunos elementos en el suelo, especialmente el nitrógeno (Hountin et al. 1997).

En cuanto al efecto de la profundidad solo se observaron diferencias significativas en el caso del nitrógeno total $(p=0,0113)$, con mayores contenidos, como es lógico, en los primeros cinco $\mathrm{cm}$. Los valores obtenidos indicaron, en la primera profundidad de muestreo, modificaciones en la proporción entre los contenidos de distintas formas de nitrógeno; así, el $\mathrm{NH}_{4}$ se modificó en $1,8 \%$ respecto al NT, en el lote con cerdos versus $2,3 \%$ en el lote sin cerdos. La proporción de $\mathrm{NO}_{3}$ en cambio no fue afectada, 1,9\% en ambos casos. Para el muestreo a mayor profundidad las proporciones no variaron entre tratamientos, los valores se ubicaron en un promedio de $2,5 \%$. Esto indica que la relación $\mathrm{NH}_{4} / \mathrm{NO}_{3}$ en la primera profundidad estuvo alrededor de 1,18 en el lote sin cerdo y 0,9 en el lote con cerdos y una relación similar, alrededor de uno en ambos casos para la segunda profundidad. La ausencia de diferencias significativas, entre las variables evaluadas, podría estar asociada a la producción de volúmenes importantes de amoníaco, a partir de las excretas, y por ende su escasa incorporación al nitrógeno del suelo, aún cuando el pH (Tabla 3) no superó el valor de 6,6 para la primera profundidad y 7,2 para la segunda. Al respecto, Vanderholm (1975), señaló que la volatilización de amonio es el principal proceso responsable de pérdidas de $\mathrm{N}$, y que puede alcanzar hasta un $65 \%$ del nitrógeno contenido en las excretas. Carey et al. (1997) y Mkhabela et al. (2009) por su parte indican que esto sucede también cuando en lugar de las excretas se añaden los purines de cerdo, y señala que la razón estaría en la producción de elevados niveles de desnitrificación por efecto de los elevados contenidos de amonio en estos materiales.

Tabla 2. Efecto del pastoreo de cerdos a campo sobre el contenido de Nitrógeno.

\begin{tabular}{cccc}
\hline Variable & Profundidad $(\mathbf{c m})$ & Sin cerdos g.kg-1 & Con cerdos g.kg-1 \\
\hline \multirow{2}{*}{$\mathrm{NT}$} & $0-5$ & $2,190 \mathrm{a}$ & $2,352 \mathrm{a}$ \\
& $5-10$ & $1,716 \mathrm{a}$ & $1,873 \mathrm{a}$ \\
$\mathrm{N}^{-N H} \mathrm{NH}_{4}$ & $0-5$ & $0,051 \mathrm{a}$ & $0,044 \mathrm{a}$ \\
& $5-10$ & $0,050 \mathrm{a}$ & $0,047 \mathrm{a}$ \\
$\mathrm{N}^{-N O}$ & $0-5$ & $0,043 \mathrm{a}$ & $0,047 \mathrm{a}$ \\
& $5-10$ & $0,046 \mathrm{a}$ & $0,046 \mathrm{a}$ \\
$\mathrm{N}-$ Orgánico & $0-5$ & $2,096 \mathrm{a}$ & $2,261 \mathrm{a}$ \\
& $5-10$ & $1,620 \mathrm{a}$ & $1,780 \mathrm{a}$ \\
\hline
\end{tabular}

Letras iguales, en las filas, indican ausencia de diferencias estadísticas 
TEMAS AGRARIOS - Vol. 18:(1) Enero - Junio 2013 (23 - 33)

Tabla 3. Variación del pH del suelo por efecto del pastoreo de cerdos.

\begin{tabular}{cccc}
\hline Variable & Profundidad $(\mathbf{c m})$ & Sin cerdos & Con cerdos \\
\hline $\mathrm{pH}$ & $0-5$ & 5,9 & 6,6 \\
& $5-10$ & 6,0 & 7,2 \\
\hline
\end{tabular}

Evaluación de la materia orgánica. En el carbono orgánico total oxidable (COT) y carbono extraíble total (CET) se observaron diferencias estadísticas significativas ( $p$ $=0,0371)$. En ambos casos hubo una disminución del COT con la profundidad, las mayores concentraciones se mantuvieron en el tratamiento con cerdos (Tabla 4).

Esta disminución ha sido vinculada a incrementos de los procesos de mineralización de algunas fracciones de la materia orgánica como consecuencia del incremento de la actividad microbiana generado por la adición de purines de cerdo (Häni et al. 1996), lo cual sería esencialmente similar en el caso del aporte directo de excretas. Los niveles de CET obtenidos (Tabla 4) mostraron que en el tratamiento sin cerdos los valores fueron mayores y significativamente diferentes, para ambas profundidades. Sin embargo en ambos casos se observó una baja proporción de CET con respecto al COT. Esto indicaría una alta proporción de sustancias en la fracción correspondiente a las huminas en este suelo.

Los contenidos de CAH y el CAF no presentaron diferencias significativas en la primera profundidad (Tabla 5). Para la segunda profundidad se observó un valor significativamente mayor $(p<0,05)$ en el tratamiento con cerdos. $\mathrm{Al}$ respecto, Plaza et al. (2003) indican que la aplicación de purines de cerdos modificó el grado de humificación, luego de tres años de tratamiento. En el caso del CAF se obtuvieron mayores valores en el tratamiento con cerdos para la primera profundidad lo cual se modificó con la profundidad, resultados similares fueron señalados por Plaza et al. (2003) para la aplicación de purines líquidos de cerdos, atribuidos a la presencia de compuestos estructuralmente similares a esta fracción de la materia orgánica. Para el CSNH las mayores concentraciones correspondieron al tratamiento con cerdos, en ambas profundidades (Tabla 5), lo cual pudiera ser consecuencia de los niveles de compuestos orgánicos parcialmente degradados presentes en las excretas.

Evaluación del fósforo. En la cuantificación de las fracciones de fósforo consideradas de fácil disponibilidad (Tabla 6) se observó que este suelo es muy pobre en este elemento. En la fracción soluble (P-resina) se encontraron valores de $\mathrm{P}$ que oscilaron entre 1,8 y 13,1

Tabla 4. Carbono orgánico total y carbono total extraíble del suelo por efecto del pastoreo de cerdos a campo.

\begin{tabular}{cccc}
\hline Variable & Profundidad $(\mathbf{c m})$ & Sin Cerdos mg.g $^{-1}$ & Con Cerdos mg.g $^{-1}$ \\
\hline \multirow{2}{*}{ COT } & $0-5$ & $3,2300 \mathrm{~b}$ & $4,3340 \mathrm{a}$ \\
& $5-10$ & $3,0730 \mathrm{~b}$ & $3,7030 \mathrm{a}$ \\
CET & $0-5$ & $0,8025 \mathrm{a}$ & $0,6409 \mathrm{~b}$ \\
& $5-10$ & $0,6769 \mathrm{a}$ & $0,4383 \mathrm{~b}$ \\
\hline
\end{tabular}

COT: Carbono orgánico total; CET: Carbono extraíble total. Letras iguales, en las filas, indican ausencia de diferencias estadísticas. 
Rivero et al. - Pastoreo de cerdos a campo

Tabla 5. Contenido de Carbono en ácidos húmicos, fúlvicos y sustancias no húmicas del suelo.

\begin{tabular}{cccc}
\hline Variable & Profundidad $(\mathbf{c m})$ & Sin Cerdos mg. $^{-1}$ & Con Cerdos mg. $^{-1}$ \\
\hline \multirow{2}{*}{ CAH } & $0-5$ & $0,077 \mathrm{a}$ & $0,074 \mathrm{a}$ \\
& $5-10$ & $0,076 \mathrm{~b}$ & $0,082 \mathrm{a}$ \\
CAF & $0-5$ & $0,029 \mathrm{~b}$ & $0,034 \mathrm{a}$ \\
& $5-10$ & $0,032 \mathrm{a}$ & $0,023 \mathrm{~b}$ \\
CSNH & $0-5$ & $0,170 \mathrm{~b}$ & $0,424 \mathrm{a}$ \\
& $5-10$ & $0,170 \mathrm{~b}$ & $0,453 \mathrm{a}$ \\
\hline
\end{tabular}

CAH: Carbono en ácidos húmicos; CAF: Carbono en ácidos fúlvicos; CSNH: Carbono en las sustancias no húmicas. Letras iguales, en las filas, indican ausencia de diferencias estadísticas.

Tabla 6. Concentraciones finales de las fracciones de fósforo en el suelo por efecto de pastoreo.

\begin{tabular}{cccc}
\hline Variable & Profundidad $(\mathbf{c m})$ & Sin Cerdos $\mathbf{~ m g . k g - 1}$ & Con Cerdos mg.kg-1 $^{-1}$ \\
\hline \multirow{2}{*}{ P- membrana } & $0-5$ & $1,8 \mathrm{~b}$ & $13,1 \mathrm{a}$ \\
& $5-10$ & $3,1 \mathrm{~b}$ & $12,3 \mathrm{a}$ \\
Pi-NaHCO3 & $0-5$ & $26,8 \mathrm{a}$ & $21,4 \mathrm{~b}$ \\
& $5-10$ & $26,6 \mathrm{a}$ & $21,0 \mathrm{~b}$ \\
Pi-NaOH & $0-5$ & $32,9 \mathrm{~b}$ & $76,9 \mathrm{a}$ \\
& $5-10$ & $19,4 \mathrm{~b}$ & $77,1 \mathrm{a}$ \\
Pi-HCl & $0-5$ & $28,6 \mathrm{a}$ & $42,3 \mathrm{a}$ \\
& $5-10$ & $32,3 \mathrm{a}$ & $42,0 \mathrm{a}$ \\
P-residual & $0-5$ & $10,0 \mathrm{a}$ & $14,7 \mathrm{a}$ \\
& $5-10$ & $10,7 \mathrm{a}$ & $14,3 \mathrm{a}$ \\
P-total & $0-5$ & $100,1 \mathrm{a}$ & $168,6 \mathrm{~b}$ \\
\hline
\end{tabular}

CAH: Carbono en ácidos húmicos; CAF: Carbono en ácidos fúlvicos; CSNH: Carbono en las sustancias no húmicas. Letras iguales, en las filas, indican ausencia de diferencias estadísticas.

$\mathrm{mg} \cdot \mathrm{kg}^{-1}$. El lote con cerdos se diferenció significativamente $(p<0,05)$ del lote sin cerdos. Las mayores concentraciones observadas en este caso podrían estar vinculadas al aporte de P-orgánico proveniente de las heces fecales. En cuanto a las profundidades no se observaron diferencias significativas. Si se comparan estos valores con los mencionados por Siso (2007) en Molisoles venezolanos, se observa que el lote sin cerdos está por debajo de este valor reportado por este autor, pero el lote con cerdos pose un valor superior, lo que indica que posiblemente, los cerdos podrían estar influenciando esta fracción de fósforo.
El P-NaHCO ${ }_{3}$, fósforo adsorbido a los coloides, fue significativamente diferente entre los lotes con y sin cerdos $(p<0,05)$, pero no hubo diferencia entre las profundidades del mismo tratamiento; los mayores valores correspondieron al lote sin cerdos. Ello podría indicar un efecto negativo de la presencia de los cerdos sobre esta fracción, vinculado a los cambios de $\mathrm{pH}$ (Tabla 3).

Con respecto a los resultados obtenidos para las fracciones de mayor recalcitrancia (Tabla 6), se pudo notar que para el $\mathrm{P}-\mathrm{NaOH}$, fósforo quimio-adsorbido a los compuestos de hierro 
y aluminio, la presencia de los cerdos indujo valores significativamente más altos, efecto que no se observó en las profundidades. No pareciera que los factores afectados por la presencia de los cerdos como el $\mathrm{pH}$ y la materia orgánica puedan explicar estos resultados. De acuerdo a la información aportada por Márquez (1989) se cree que esto sería derivado de la variabilidad mineralógica del área como producto de la confluencia de las formaciones las Mercedes y Las Brisas.

El P-HCl, es decir, el unido al calcio fue más bajo que el $\mathrm{P}$ unido al hierro y al aluminio; esto se explicaría con base en que los materiales cálcicos no son dominantes en estos suelos, por lo tanto la fijación en forma cálcica es mucho menor que la producida para el hierro y el aluminio, esto a pesar de los valores de $\mathrm{pH}$ (Tabla 3). Para esta fracción no hubo efecto ni de la presencia de cerdos ni de las profundidades.

El fósforo residual, extraído con $\mathrm{HClO} 4-\mathrm{HNO} 3$ no mostró diferencias ni para el manejo bajo cerdos ni para las profundidades. Los valores encontrados en el tratamiento sin cerdos fueron 10 y 10,7 mg. $\mathrm{kg}^{-1}$ de $\mathrm{P}$, mientras que para el tratamiento con cerdos fue de $14,7 \mathrm{y}$ 14,3 mg. $\mathrm{kg}^{-1}$ de $\mathrm{P}$, para las profundidades $0-5 \mathrm{y}$ 5-10 cm, respectivamente. Resulta interesante destacar que en este suelo las concentraciones de fósforo en esta fracción resultan bastante inferiores a los mencionados para otros molisoles venezolanos (Siso 2007), lo cual también pudiera atribuirse de la influencia de la variabilidad en la mineralogía del área de estudio, descrita por Márquez (1989).

En la evaluación del fósforo total (Tabla 6), se observó un efecto significativo del manejo bajo cerdos y al igual que en las demás fracciones no se observó efecto de las profundidades. La diferencia encontrada entre los tratamiento se debe a la influencia de los aportes de los cerdos sobre el contenido de fósforo en el suelo por sus aporte de excretas sumado a los desperdicios que se producen en su alimentación.

\section{CONCLUSIONES}

El tiempo durante el cual fueron aplicados los tratamientos no permitieron evidenciar el efecto del sistema de producción de cerdos a campo sobre el nitrógeno total o sus formas amoniacales o nítricas, como tampoco el carbono de los ácidos húmicos y fúlvicos, pero influyeron en el carbono orgánico extraíble y en el de las sustancias no húmicas. Para el caso del fósforo se observó un incremento generalizado de las distintas fracciones evaluadas, especialmente del fósforo extraíble en resina.

\section{AGRADECIMIENTOS}

Los autores agradecen el apoyo financiero del Consejo de Desarrollo Científico y Humanístico de la Universidad Central de Venezuela $(\mathrm{CDCH})$ a través del proyecto de investigación № PG-01-00-7143-2008, titulado "Impacto del Manejo de Sistemas de Producción de Cerdos a Campo sobre el Suelo".

\section{REFERENCIAS}

Bremner, J. 1965. Methods of Soil Analysis. Part 2. Chemical and Microbiological Properties, 20 eds C.A. Black, D.D. Evans, L.E. Ensminger \& F.E. Clark. Am. Soc. of Agron. Madison, WI, p1324-1345. 
Bremner, J. 1996. Nitrogen-total. In: Methods of Soil Analyses, Part 3 Chemical Methods. ASA, INC. SSS America, INC. Publisher. Wisconsin, USA, p1085-1121.

Buehler, S., Oberson, A., Rao, M., Friesen, K. y Frossarda, E. 2002. Sequential phosphorus extraction of a 33P-Labeled Oxisol under contrasting agricultural systems. Soil Sci. Soc. Am. J. 66:868-877.

Campagna, D.;Somenzini,D.2005. Elementos a tener en cuenta para decidir que categorías confinar en los sistemas de producción porcina a campo para mejorar su eficiencia. FERICERDO. Marcos Juárez, 19 y 20 de agosto 2005.

Carey, L., Rate, A. y Cameron, K. 1997. Fateof nitrogen in pig slurry applied to a New Zealand pasture soil. Australian J. Soil Res. 35(4):941-959.

Ciavatta,C.,Govi,M., Vittori,L.ySequi,P.1990. Characterization of humified compounds by extraction and fractionation on solid polyvinylpyrrolidone. J. Chrom. 643:261-270.

Dalla, O. 1998. Sistema intensivo de Suinos criados ao $\mathrm{Ar}$ livre-Siscal: Manejo, índices de produtividade, custo de implatacao e prducao- Embrapa-Cnpsa. I Encuentro de producción animal de cerdos a campo. On-line: www.sian. info.ve/porcinos/ [Marzo de 2011].

Díaz, I. y Egaña, I. 2000. Manejo y utilización de los desechos fecales porcinos: una alternativa como recurso alimentario para rumiantes. Santiago, Chile. U. Chile, Fac. Cs. Veterinarias y Pecuarias, Depto. Fomento Producción Animal. Serie Apuntes Docentes № 19, p45.

González, C. 1999. Potencialidad del cerdo criollo y la producción alternativa de cerdosen Venezuela. Universidad Central de Venezuela. Facultad de Agronomía. Instituto de Producción Animal, Maracay - Estado Aragua. Mimeografiado, p5.

Häni, H., Siegenthaler, A. y Candinas, T. 1996. Soil effects due to sewage sludge application in agriculture. Fertilizer Research 43:149-156.

Heanes, D. 1984. Determination of total organic-C in soil by an improved chromic acid digestión and spectrophotometric procedure. Com. Soil Sci. Plant Anal. 15:1191-1213.

Hedley,J.,Stewart,J.yChauhan,B.1982.Changes in inorganic and organic phosphorus fractions induced by cultivation practices and by laboratory incubations. Soil Sci. Soc. Am. J. 46:970-976.

Hountin, A., Couillard, D. y Karam, A. 1997. Soil carbon, nitrogen and phosphorous contents in maize plots after 14 years of pig slurry applications. J. Agric. Sci. 129:187-191.

Juárez, J., Rivero, C. y Rodríguez, J. 2004. Efecto del uso de coberturas sobre las fracciones y disponibilidad del fósforo del suelo. Venesuelos 12:16-24. 
Maag, M. y Vinther, F. 1999. Effect of temperature and water on gaseous emissions from soils treated with animal manure. Soil Sci. Soc. Am. J. 63:858-865

Márquez, O. 1989. Caracterización mineralógica y génesis de suelos de dos ambientes geoquímicos de la cuenca del Rio Güey. Trabajo de Grado, Postgrado en Ciencia del Suelo. Universidad Central de Venezuela, p200.

Mkhabela, S., Gordon, R., Burton, D., Smith, E. y Madani, A. 2009. The impact of management practices and meteorological conditions on ammonia and nitrous oxide emissions following application of hog slurry to forage grass in Nova Scotia. Agriculture, Ecosystems \& Environ 130:41-49.

Murphy, J. y Riley, P. 1962. A modified single solution method for the determination of phosphate in natural waters. Anal Chim. Acta 27:31-36.

Navarro, S. y Navarro, G. 2000. Química agrícola. Madrid: Mundiprensa, p760.

Olivas, L. y Sequeira, C. 2002. Evaluación de diferentes efluentes de cerdo como bioabono sobre el crecimiento y el rendimiento del cultivo de sorgo (Sorghum bicolor L. Moench) y las propiedades químicas del suelo. Tesis de Grado, Universidad Nacional Agraria, Managua, Nicaragua.

Plaza, C., Senesi, N., Polo, A., Brunetti, G., García-Gil, J. y D'orazio, V. 2003. Soil fulvic acid properties as a means to assess the use of pig slurry amendment. Soil Till. Res. 74(2):179-190.

Rivero, C., Senesi, N., Paolini, J.y D’Orazio, V. 1998. Characteristics of humic acids of some Venezuelan soils. Geoderma 81(3-4):227-239.

Rochette, P., Angers, D. y Côté, D. 2000. Soil carbon and nitrogen dynamics following application of pig slurry for the 19th consecutive year: I. Carbon dioxide fluxes and microbial biomass carbon. Soil Sci. Soc. Am. J. 64:1389-1395.

Rodríguez, M., Pulido, M., Rey, J., Lobo, D., Araque, H. y Rivero, C. 2010. Efecto del pisoteo en sistemas de producción de cerdos a campo sobre algunas propiedades del suelo. Agronomía Tropical, 60:119-130.

Salas,A. 2001. Evaluacióndelaprocedenciadel fósforo absorbido por el maíz en mezcla de roca fosfórica Riecito y superfosfato triple en un Ultisol bajo condiciones de invernadero. Trabajo de Ascenso a Profesor Agregado. Universidad Central de Venezuela, p28.

Siso, W. 2007. Estudio del fósforo en algunos suelos venezolanos. Trabajo de Grado. Facultad de Agronomía. Universidad Central de Venezuela, p54.

Sosa, O. 2005. Los estiércoles y su uso como enmiendas orgánicas. On-line: www.fcagr. unr.edu.ar/Extension/ [Febrero 2011]. 
Ternicier,C.2005.Planteamientodeunmodelo teórico de indicadores para la evaluación del impacto ambiental de sistemas intensivos de producción de carne porcina de exportación. Universidad Católica de Temuco. Chile, p86.

Tiessen, H. y Moir, O. 1993. Characterization of available $\mathrm{P}$ by sequential extraction. In: Carter, M. R. (ed.). Soil Sampling and Methods of Analysis. Tesis de Maestría, Boca Ratón.
Vanderholm, H. 1975. Nutrient losses from livestock waste during storages treatment and handling. 3rd Symposium on Livestock Waste Management. Chicago, p20.

Wodzinski, J. y Ullah, A. 1996. Phytases. Advances in Applied Microbiology 42:263-302. 